\title{
Relationship of Off-Pump Coronary Artery Bypass Surgery with the Cardiac Enzyme Troponin T, LVEF, TAPSE and Post-Operative Recovery
}

\author{
Jayita Chakrabarti1 ${ }^{1}$, Subhendu Sekhar Mahapatra ${ }^{2}$, Ratan Mondal ${ }^{3}$ \\ 1Department of Cardiothoracic and Vascular Surgery, IPGME\&R, SSKM Hospital, (The West Bengal University of \\ Health Sciences), Kolkata, West Bengal, India. ${ }^{2}$ Department of Cardiothoracic and Vascular Surgery, IPGME\&R, \\ SSKM Hospital, (The West Bengal University of Health Sciences), Kolkata, West Bengal, India. ${ }^{3}$ Department of \\ Cardiothoracic and Vascular Surgery, Nightingale Hospital, Kolkata, West Bengal, India.
}

\section{ABSTRACT}

\section{BACKGROUND}

Cardiovascular diseases (CVD) are the leading cause of death globally including India. Ischemic heart disease is the most common cause of death. There is a sharp rise in Off Pump Coronary Artery Bypass Surgery (OPCAB), which increases cardiac enzymes like Troponin $\mathrm{T}$, that is used as prognostic guide. In triple vessel disease 40 to $50 \%$ cases involve right ventricle, therefore along with left ventricular ejection fraction (LVEF), Tricuspid Annular Plane Systolic Excursion (TAPSE) can be used as a prognostic guide, which can be measured by noninvasive doppler echocardiography.

\section{METHODS}

Patients with specific criteria who underwent OPCAB surgery from January 2018 to March 2019 were studied. Both pre- and post-operative (14-16 hours) Troponin T (TropT) sensitivity tests were done and only preoperative Trop T negative patients were selected for the study. Postoperative patients were divided into 2 groups, TropT positive and negative. Echocardiography was done on the day before surgery, on postoperative day 5 (POD5) and postoperative day 30 (POD30). Results were compared between the two groups.

\section{RESULTS}

Among 88 patients thus selected, 34 patients (38.63\%) were Trop T positive, and 54 patients (61.37\%) were Trop T negative. On post-operative day 5, increase in brain natriuretic peptide (BNP), decrease in TAPSE and decrease in haemoglobin were significant in Trop T positive group. Need for ventilation and postoperative hospital stay were significantly prolonged in positive groups. In POD30, decrease in albumin, decrease in LVEF and TAPSE all are significant in TropT positive group.

\section{CONCLUSIONS}

It is important to determine the prognosis of OPCAB patients in early postoperative period. Troponin $\mathrm{T}$ has a significant prognostic value in OPCAB patients supported by LVEF and TAPSE.

\section{KEY WORDS}

Triple Vessel Disease, OPCAB, Troponin T, LVEF, TAPSE, Outcome
Corresponding Author: Subhendu Sekhar Mahapatra, Department of CTVS, SSKM Hospital, \#244, AJC Bose Road, Kolkata-700020, West Bengal, India.

E-mail: subhendu.mahapatra1972@gmail.com

DOI: $10.14260 / \mathrm{jemds} / 2020 / 146$

Financial or Other Competing Interests: None.

How to Cite This Article:

Chakrabarti J, Mahapatra SS, Mondal R. Relationship of off-pump coronary artery bypass surgery with the cardiac enzyme troponin T, LVEF, TAPSE and postoperative recovery. J. Evolution Med. Dent. Sci.2020;9(09):673-677, DOI: 10.14260/jemds/2020/146

Submission 03-12-2019, Peer Review 04-02-2020, Acceptance 11-02-2020, Published 02-03-2020.

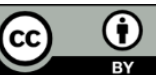




\section{BACKGROUND}

Cardiovascular diseases (CVD) are the leading cause of disease burden and deaths globally.(1) The United Nations, alarmed about the rising burden of non-communicable diseases(NCDs) in middle income group and low income group countries and has acknowledged that this is one of the major threats to sustainable developments in $21^{\text {st }}$ century. World Health Organisation subsequently developed targets for prevention and control of NCDs in 2013. Cardiovascular diseases (CVD) makes a large part of NCDs.(1,2) In National Health Policy 2017, India aims to reduce premature deaths from CVDs, especially ischaemic heart disease to $25 \%$ by 2025 . Our country with 1.3 billion people with significant cultural and lifestyle diversities is going through a varied epidemiological transition regarding the trend of ischaemic heart disease among its different states from 1990 to 2016. Cardiovascular disease prevalence has increased in India from 25.7 million in 1990 to 54.5 million in 2016 and there is nearly $46 \%$ rise in deaths due to CVDs especially with ischaemic heart disease. This trend is highest in Kerala, Punjab, Tamilnadu and followed by Andhra Pradesh, Himachal Pradesh, Maharashtra, Goa and West Bengal.(1,2)

Our centre, IPGME\&R and SSKM Hospital, is not only a high-volume tertiary care hospital in West Bengal, it is the oldest cardiac surgery training centre in eastern India. It is observed that there is a very sharp rise in number of patients with triple vessel coronary artery disease, admitted for coronary artery bypass grafting surgery (CABG) in past few years. As in other centres in India, in our centre also off pump coronary artery bypass surgery (OPCAB) highly outnumbers on pump coronary artery bypass surgeries (ONCAB).

It is known that biomarker-measurement can predict outcome after cardiac surgery but obviously every surgical procedure is not associated with same degree of rise of cardiac biomarkers. OPCABs are very often associated with rise of such markers postoperatively. Some literatures suggest cardiac troponin $\mathrm{T}$ (Trop-T) measurement may be superior for the outcome prediction than MB isoenzyme of creatine kinase (CPK-MB) and even cardiac troponin I (Trop-I).(3) Trop-T elevation has repeatedly and unequivocally been shown to be prognostic for delayed recovery and longer intensive cardiac care unit (ICU) stay. But assessing prognosis after CABG only with cardiac enzymes like Troponins is not always easy. $(4,5,6)$ Measurement of Left Ventricular Ejection Fraction (LVEF) has also a prognostic value after OPCAB but is not always a suitable guide, especially in patients with triple vessel disease with inferior wall ischaemia and right coronary artery dominance. Thus, we added Tricuspid Annular Plane Systolic Excursion (TAPSE) in evaluation of post-operative OPCAB patients for augmentation of prognostic value regarding recovery. Studies have shown TAPSE has a good correlation with ECG evidence of right ventricular (RV) infarction, as well as, an independent predictor of mortality in inferior wall myocardial infarction. It has also been found to have a good correlation with radionuclide derived EF. Studies have shown TAPSE $\leq 16 \mathrm{~mm}$ predicted proximal RCA lesion with a sensitivity of $92.3 \%$ and specificity of $100 \%$.

Our centre has a wide drainage area spanning different states of eastern India. No study has been done yet on postoperative recovery of $\mathrm{OPCAB}$ patients of this demographic area combining estimation of three parameters Trop-T, LVEF and TAPSE for prognostic value.

\section{METHODS}

From January 2018 to March 2019, patients who underwent OPCAB in IPGME\&R and SSKM Hospital were studied. Patients were selected depending upon the specific selection criteria. Among all the studied patients, 88 patients were found to meet the below-mentioned selection criteria and enrolled in this retrospective study. As this is a retrospective record-based study, our Institutional Ethics Committee has granted our request for waiver of the written informed consent for the study.

\section{Patient Selection Criteria}

1. Only male patients with diagnosed triple vessel disease involving left, right coronary arteries and circumflex artery were included as causal factors and disease prognosis are different in male and female genders in ischemic heart disease.

2. Age limit- 45-65 years, the most common age group affected by ischemic heart disease in the demographic zone under study.

3. All patients selected were diabetic.

4. All patients were on atorvastatin, aspirin, clopidogrel and metoprolol tablets preoperatively and clopidogrel and aspirin were stopped five to seven days before operation.

5. All patients were normotensive before operation, as uncontrolled hypertension affects recovery significantly, may cause encephalopathy or stroke postoperatively.

6. All patients were Troponin T negative preoperatively.

7. Left ventricular ejection fraction was more than $45 \%$ and with such ejection fraction hemodynamic stability during operation would be better maintained.

8. Patients with TAPSE more than $16 \mathrm{~mm}$ were included (as $16 \mathrm{~mm}$ is the consensus cut-off value for coronary artery disease).

9. Patients with stents in RCA were excluded as this can affect preoperative TAPSE level.

10. Patients were screened for serum Thyroid Stimulating Hormone (TSH) and free T4 level. Only those patients with normal thyroid profile were selected as disturbance in thyroid function affects postoperative recovery.

11. No patient had other associated co morbidities like chronic kidney, lung diseases, liver and peripheral arterial diseases.

12. All patients had 4 grafts - as number of grafting is the function of time and may influence ischemic time.

13. The preop NYHA (New York Heart Association classification for heart failure) class was either III or IV in all patients.

All patients had preoperative (preop) Trop T sensitivity test and routine echo evaluation was done including LVEF and TAPSE level measured, the day before surgery. Trop $\mathrm{T}$ sensitivity test was again done on post-operative day (POD)- 1 morning i.e., after 14-16 hours after surgery. All patients were postoperatively evaluated with 2D Echocardiography and colour Doppler study on POD 5. We use to discharge our post op OPCAB patients with uneventful and smooth recovery on POD 5. By protocol routine echo assessment was done along with LVEF and TAPSE measurement in the postoperative second visit in day -30. All patients (pts) underwent operation, with general anaesthesia, intubation and midline sternotomy, 
under same anaesthetic and surgical team. All patients received LIMA to LAD grafting, RSVG to one diagonal artery (sequential), one obtuse marginal (sequential) and one PDA/distal RCA grafting (end anastomosis). Suction stabiliser, deep pericardial stays and gauge packing were used in all patients.

All patients received infusion nitroglycerin and/or dobutamine, low dose of noradrenaline and diuretic therapy peroperative and postoperatively. Reopening was done in two patients in ICU due to bleeding issues. Postoperatively 12 patients had signs and symptoms of heart failure, mild to moderate in presentation, with variable incidence of increased heart rate, pedal oedema, rales at the base of the lungs, night cough, distress on lying down, anorexia but all of them were treated successfully. Respiratory complications included postop prolonged mechanical ventilation (more than 36 hours) and one patient needed tracheostomy and recovered late, one patient had CVA of ischaemic variety involving area supplied by posterior cerebral artery, recovered and discharged from hospital after six weeks post operatively. Wound infection, superficial soft-tissue infection, occurred in 6 pts, 3 of them needed sternal wire removal after two months of surgery.

\section{Statistical Analysis}

For statistical analysis, data were entered into a Microsoft excel spreadsheet and then analyzed by SPSS (version 25.0; SPSS Inc., Chicago, IL, USA). Data had been summarized as mean and standard deviation for numerical variables and count and percentages for categorical variables. Two-sample t-tests for a difference in mean involved independent samples or unpaired samples. p-value $\leq 0.05$ was considered for statistical significance.

\section{RESULTS}

Among 88 pts, 34 (38.63\%) pts, were positive with Trop T sensitivity test post operatively and the rest 54 pts, (61.36\%) were negative for the same. There were 16 patients $(18.18 \%)$ with LVEF $<35 \%$ in Trop $\mathrm{T}$ (+ve) group and 9 patients $(10.22 \%)$ in Trop T (-ve) group. TAPSE were $<14$ in 7 patients $(7.95 \%)$ in Trop $\mathrm{T}(+\mathrm{ve})$ group and 6 patients $(6.81 \%)$ in Trop $\mathrm{T}$ (-ve) group. Table 1 compares the preoperative characteristics of patients belonging to two groups with postoperative Trop T (+ve) and Trop T (-ve) results. Age, height, renal function, nutritional status along with left ventricular function and TAPSE were not significantly different between these two groups. Body wt, BMI, Blood pressure status were also not different.

In Table 2 early postoperative routine investigations and in- hospital outcomes are depicted. Estimated BNP and $\mathrm{Hb}$ values were significantly different in two groups. LVEF was not different $(p=0.06)$ but difference is significant in TAPSE $(p=0.01)$ measurement in POD 5. Measured albumin level, PRBC and FFP transfusion were not significantly different post operatively though 12 patients had developed signs and symptoms of heart failure in early postoperative phase. The need for prolonged ventilation ( $>36 \mathrm{hrs}$.) and postoperative hospital stay were significantly different in two said groups $(p=<0.0001)$. In Table 3 shows estimated values of parameters signifying heart failure but in POD 30, albumin, $\mathrm{Hb}$ and BNP were not significantly different but LVEF and TAPSE were. $(\mathrm{p}=<0.0001)$.

\begin{tabular}{|c|c|c|c|}
\hline Characteristics & $\begin{array}{c}\text { Trop-T (+ve) } \\
\text { (n=34) }\end{array}$ & $\begin{array}{c}\text { Trop-T (-ve) } \\
(\mathbf{n}=54)\end{array}$ & $\mathbf{p}$ \\
\hline Age (years) & $54.75 \pm 9.90$ & $56.04 \pm 6.32$ & 0.45 \\
\hline Height (metres) & $1.62 \pm 0.05$ & $1.60 \pm 0.05$ & 0.07 \\
\hline Body weight (Kg) & $56.49 \pm 7.38$ & $55.20 \pm 7.09$ & 0.41 \\
\hline BMI (Kg/m²) & $20.37 \pm 9.30$ & $21.63 \pm 2.15$ & 0.34 \\
\hline Systolic Blood Pressure (mmHg) & $123.75 \pm 17.19$ & $126.15 \pm 13.34$ & 0.46 \\
\hline Diastolic Blood Pressure (mmHg) & $84.25 \pm 4.79$ & $79.50 \pm 9.32$ & 0.54 \\
\hline Creatinine (mg/dl) & $1.29 \pm 0.70$ & $1.32 \pm 0.30$ & 0.78 \\
\hline Albumin (gm/dl) & $3.1 \pm 1.1$ & $3.0 \pm 1.20$ & 0.69 \\
\hline Haemoglobin (gm/dl) & $11.42 \pm 1.30$ & $10.97 \pm 1.96$ & 0.23 \\
\hline ALT(units/L) & $51.33 \pm 19.22$ & $49.60 \pm 17.0$ & 0.65 \\
\hline AST (units/L) & $44.00 \pm 22.80$ & $33.13 \pm 17.70$ & 0.84 \\
\hline LVEF (\%) & $48.00 \pm 2.50$ & $45.50 \pm 3.78$ & 0.21 \\
\hline TAPSE (mm) & $16.02 \pm 0.24$ & $15.97 \pm 1.03$ & 0.78 \\
\hline Table 1. Comparative Pre-Op Data for Two Groups with \\
Post-Op Trop-T (+ve) vs Trop -T (-ve) Results \\
\hline \multicolumn{4}{|l}{} \\
\hline
\end{tabular}

\begin{tabular}{|c|c|c|c|}
\hline Characteristics & $\begin{array}{c}\text { Trop-T (+ve) } \\
\text { (n=34) }\end{array}$ & $\begin{array}{c}\text { Trop-T (-ve) } \\
\text { (n=54) }\end{array}$ & p \\
\hline Haemoglobin (gm/dl) & $8.32 \pm 2.43$ & $9.46 \pm 1.50$ & 0.007 \\
\hline Albumin (gm/dl) & $2.60 \pm 1.0$ & $2.78 \pm 1.66$ & 0.57 \\
\hline BNP (pg/ml) & $1054.79 \pm 684.69$ & $672.34 \pm 229.98$ & 0.0003 \\
\hline LVEF (\%) & $37.67 \pm 6.62$ & $42.05 \pm 4.26$ & 0.06 \\
\hline TAPSE (mm) & $13.36 \pm 1.02$ & $14.04 \pm 1.32$ & 0.012 \\
\hline PRBC Transfusion (units) & $3.20 \pm 1.76$ & $3.0 \pm 1.23$ & 0.53 \\
\hline FFP Transfusion (ml) & $586.9 \pm 646.90$ & $540.0 \pm 560.15$ & 0.72 \\
\hline Postop Hospital Stay (days) & $7 \pm 1.25$ & $4.6 \pm 2.3$ & $<0.0001$ \\
\hline Ventilator Support (hours) & $32.37 \pm 4.75$ & $17.15 \pm 5.50$ & $<0.0001$ \\
\hline
\end{tabular}

Table 2. Comparative Data of Post-Op Parameters between the Two Groups with Post-Op Trop-T (+ve) vs Trop-T (-ve) Results in POD 5

\begin{tabular}{|c|c|c|c|}
\hline Characteristics & Trop-T (+ve)(n=34) & Trop-T (-ve)(n=54) & p \\
\hline Haemoglobin (gm/dl) & $9.17 \pm 2.34$ & $9.69 \pm 1.92$ & 0.26 \\
\hline Albumin (gm/dl) & $2.91 \pm 0.36$ & $3.01 \pm 0.20$ & 0.09 \\
\hline BNP (pg/ml) & $544.0 \pm 270.26$ & $620.0 \pm 300.92$ & 0.23 \\
\hline LVEF (\%) & $43.34 \pm 0.02$ & $46.32 \pm 3.7$ & $<0.0001$ \\
\hline TAPSE (mm) & $14.10 \pm 0.29$ & $15.30 \pm 0.19$ & $<0.0001$ \\
\hline Table 3. Comparative Data of Post-Op Parameters between the Two \\
Groups with Post-Op Trop-T (+ve) vs Trop-T (-ve) Results in POD 30 \\
\hline
\end{tabular}

\section{DISCUSSION}

Studies related to the changing patterns of cardiovascular diseases and their risk factors, are done on different states of India over decades. They depict states of eastern India belongs to an epidemiological transition phase. There is a steep rise in Ischaemic heart disease and related deaths in recent times. ${ }^{(1,2)}$ Being a tertiary care hospital, our centre has the wide drainage area from various parts of eastern India and is experiencing very high number of OPCAB done per year. It is a known fact that OPCAB increases cardiac enzymes postoperatively, $(3,4)$ but predicting prognosis based only on this is problematic.(5) Moreover, LVEF is affected in many cases postoperatively and also can be considered as a postoperative prognostic guide,(6) but regarding surgical treatment in triple vessel disease where $70 \%$ of hearts are right coronary artery dominated,(7) considering one right ventricular parameter may give a better prognostic value. Our study is based on eastern Indian population and considered post-operative Trop T sensitivity test, LVEF\%, TAPSE measurement as the cumulative predictors in post OPCAB patients and as per literature, no such study is yet been done on such a demographic zone.

It is a well-established fact that cardiac enzymes Troponin T, Troponin I, CPK-MB elevation is nearly universal after cardiac surgical procedures like $\mathrm{OPCAB},(8,9)$ there are multiple 
mechanisms proposed to explain the finding of myocardial injury after such cardiac surgery when preoperative level is normal. Intra-operative injury may occur related to cardiac manipulation, application of stabiliser, applying diathermy near to coronary artery, prolonged grafting time, intraoperative defibrillation or acute per operative hemodynamic instability, while post-operative injury may be associated with prolonged hypotension, acute loss of by-pass grafts. $(10,11)$ Sampling at ICU within 12-24 hours postoperatively may provide unique prognostic information.(12)

In our centre TROP T Sensitive kits (Cobas -Roche) are routinely used after 12 hours of completion of OPCAB surgery and overall prognosis is assessed. The test contains two monoclonal antibodies specific for cardiac Troponin $\mathrm{T}$, one gold -labelled, the other biotinylated. Both form a sandwich complex with any cTnT present in blood sample. Erythrocytes are removed from the sample and the plasma passes through the detection zone, in which the cTnT sandwich complexes gather along a line, appearing as a red streak. Excess goldlabelled antibodies gather along the control line, signalling visually that the test was valid. The positive result means that the concentration of Trop $\mathrm{T}$ in the sample is above the test threshold value of $0.1 \mathrm{ng} / \mathrm{ml}$.(13)

It is an established fact that LVEF is an important parameter for assessing LV systolic function. Many patients, who are treated with aggressive revascularization therapy like OPCAB and medical therapy following $\mathrm{MI}$, will have an improved LV systolic function, up to $50 \%$ of patients do not demonstrate improvement in LVEF several months after their index MI.(14) The prognostic significance of the heterogeneity in LVEF change is not fully understood, yet may be important in terms of risk assessment and the routine management of post-MI patients.(15)

Moreover, acute myocardial infarction, 40\%-50\% cases involve inferior wall of the heart.(16) Approximately 30\%-50\% of patients with this inferior infarction have some involvement of the right ventricle. Right ventricular infarction almost invariably develops in association with large infarction of adjacent septum and left ventricular inferior walls.(17) 0 basis of this we added TAPSE to augment the prognostic value. Tricuspid annular plane systolic excursion is a parameter of global RV function which describes apex to base shortening. It correlates closely with RVEF, is easy to measure and is an excellent parameter for predicting poor prognosis in patients with acute inferior wall myocardial infarction.(17,18) As literature suggests, TAPSE $\leq 16 \mathrm{~mm}$ has been shown to correlate with RV systolic dysfunction in a patient population with coronary artery disease involving inferior wall and right ventricle. $(18,19)$

We hypothesised that TAPSE along with a simple index LVEF could be the cumulative prognostic indicators in post $\mathrm{OPCAB}$ patients for guiding treatment. Preoperative cardiac, renal, liver function were not different between Trop $\mathrm{T}(+\mathrm{ve})$ and Trop $\mathrm{T}$ (-ve) groups. Lung ailments were an exclusion criterion during patients' selection procedure. In immediate post-operative period some heart failure parameters were significantly different in these two groups, as well as, TAPSE but not LVEF. Time needed for mechanical ventilatory support and postoperative hospital stay $(\mathrm{p}=<.0001)$ were significantly different in two groups that is difference in overall morbidity. In second visit that is POD 30, LVEF\% and TAPSE were significantly different in two groups $(\mathrm{p}=<0.0001)$. Though 12 patients had some signs of heart failure during immediate post op period, there were no significant difference in regard with albumin level and FFP, PRBC transfusion. The likely reason may be our study sample is small.

\section{CONCLUSIONS}

In post OPCAB patients, elevation of cardiac enzyme Trop T is used as a prognostic guide especially when preoperative Trop $\mathrm{T}$ value is normal. LVEF is another well-known parameter in immediate and in early post-operative period of such patients for guiding postoperative recovery. In triple vessel disease with inferior wall ischaemia and/or infarction, there remains a heterogeneity using LVEF alone as a prognostic guide. A RV parameter TAPSE, signifying a global RV function, when added to Trop $\mathrm{T}$ and LVEF, they increase the cumulative prognostic value to a greater degree in such patients.

\section{REFERENCES}

[1] Prabhakaran D, Jeemon P, Sharma M, et al. The changing pattern of cardiovascular diseases and their risk factors in the states of India: the Global Burden of Disease Study. The Lancet 2018;6 (12):PE1339-PE51.

[2] Prabhakaran D, Jeemon P, Roy A. Cardiovascular diseases in India: current epidemiology and future directions. Circulation 2016;133 (16):1605-20.

[3] Brown JR, Hernandez F Jr, Klemperer JD, et al. Cardiac troponin $\mathrm{T}$ levels in on- and off-pump coronary artery bypass surgery. Heart Surg Forum 2007;10 (1):E42-6.

[4] Huyghe N, Bouchez S, Moerman A, et al. Cardiac troponin $\mathrm{T}$ release patterns after off-pump coronary bypass surgery. Eur J Anaesth 2013;30:58.

[5] Januzzi JL Jr. Troponin testing after cardiac surgery. HSR Proc Intensive Care Cardiovasc Anesth 2009;1 (3):22-32.

[6] Dalén M, Lund LH, Ivert T, et al. Survival after coronary artery bypass grafting in patients with preoperative heart failure and preserved vs reduced ejection fraction. JAMA Cardiol 2016;1 (5):530-8.

[7] Das H, Das G, Das DC, et al. A study of coronary dominance in the population of Assam. J Anat Soc India 2010;59 (2):187-91.

[8] Da Costa LMA, Hueb W, Nomura $\mathrm{CH}$, et al. Significant elevation of biomarkers of myocardial necrosis after coronary artery bypass grafting without myocardial infarction established assessed by cardiac magnetic resonance. Medicine (Baltimore) 2017;96 (6):e6053.

[9] Wang TK, Stewart AHR, Ramanathan T, et al. Diagnosis of MI after CABG with high-sensitivity troponin $\mathrm{T}$ and new ECG or echocardiogram changes: relationship with mortality and validation of the Universal Definition of MI. Eur Heart J Acute Cardiovasc Care 2013;2(4):323-33.

[10] Chakravarthy MR, Prabhakumar D. Anaesthesia for off pump coronary artery bypass grafting - the current concepts. Indian J Anaesth 2007;51(4):334. 
[11] Bappu NJ, Venugopal P, Bisoi AK, et al. Troponin-I release after cardiac surgery with different surgical techniques and post-operative neurological outcomes. Mcgill J Med 2006;9 (2):88-94.

[12] Stahel HTT, Do DP, Klaus JB, et al. Clinical relevance of Troponin $\mathrm{T}$ profile following cardiac surgery. Front Cardiovasc Med 2018;5:182.

[13] Koerbin G, Tate JR, Hickman PE. Analytical characteristics of the Roche highly sensitive troponin $\mathrm{T}$ assay and its application to a cardio-healthy population. Ann Clin Biochem 2010;47 (Pt 6):524-8.

[14] Reibis R, Salzwedel A, Bonaventura K, et al. Improvement of left ventricular ejection fraction in revascularized postmyocardial patients: indication for statistical fallacy. BMC Res Notes 2017;10 (1):244.

[15] Chew DS, Heikki H, Schmidt G, et al. Change in left ventricular ejection fraction following first myocardial infarction and outcome. JACC Clinical Electrophysiology 2018;4 (5):672-82.
[16] Aher M, Bansal S, Isser $H$, et al. Assessment of right ventricular function in patients with acute myocardial infarction. J Am Coll Cardiol 2018;17 (Suppl 11):10-12.

[17] Forfia PR, Fisher MR, Mathai SC, et al. Tricuspid annular displacement predicts survival in pulmonary hypertension. Am J Respir Crit Care Med 2006;174 (9):1034-41.

[18] Schmid E, Hilberath JN, Blumenstock G, et al. Tricuspid annular plane systolic excursion (TAPSE) predicts poor outcome in patients undergoing acute pulmonary embolectomy. Heart Lung Vessel 2015;7(2):151-8.

[19] Chapman AR, Adamson PD, Mills NL. Assessment and classification of patients with myocardial injury and infarction in clinical practice. Heart 2017;103 (1):10-8. 